\title{
DNA Probes Using Fluorescence Resonance Energy Transfer (FRET): Designs and Applications
}

BioTechniques 31:1106-1121 (November 2001)

\author{
Vladimir V. Didenko \\ Baylor College of Medicine, \\ Houston, TX, USA
}

\begin{abstract}
Fluorescence resonance energy transfer (FRET) is widely used in biomedical research as a reporter method. Oligonucleotides with a DNA backbone and one or several chromophore tags have found multiple applications as FRET probes. They are especially advantageous for the real-time monitoring of biochemical reactions and in vivo studies. This paper reviews the design and applications of various DNA-based probes that use FRET. The approaches used in the design of new DNA FRET probes are discussed.
\end{abstract}

\section{INTRODUCTION}

Fluorescence resonance energy transfer (FRET) is a quantum phenomenon occurring between two dye molecules. Excitation is transferred from a donor to an acceptor fluorophore through dipole-dipole interaction without the emission of a photon. As a result, the donor molecule fluorescence is quenched, and the acceptor molecule becomes excited. It then loses energy via heat or fluorescent emission, called sensitized emission. When FRET occurs, the intensity of the donor fluorescence, lifetime, and quantum efficiency decrease, and the fluorescence intensity of the acceptor increases (22-24).

Although the theory of FRET was developed by Theodor Förster in the late 1940s $(23,24)$, the first biological applications of this phenomenon started in the 1970s, dealing primarily with protein research. FRET-based DNA applications only began to appear in the mid-1980s and 1990s.

\section{THEORY OF FRET}

There are many detailed reviews describing the physical mechanisms of FRET $(9,10,13,60,79)$. We will only briefly outline its basic points.

The major expression derived by Förster for the rate of energy transfer from the excited donor to the acceptor $\left(\mathrm{k}_{\mathrm{T}}\right)$ can be given in the following form (87):

$$
\mathrm{k}_{\mathrm{T}}=\text { const } \mathrm{J} \mathrm{n}^{-4} \mathrm{R}^{-6} \mathrm{\kappa}^{2} \quad \text { [Eq. 1] }
$$

where $\mathbf{R}$ is the distance between donor and acceptor chromophores, and $\mathbf{J}$ represents the spectral overlap integral, proportional to the overlap of emission spectrum of the donor and absorption spectrum of the acceptor. The essential FRET requirement is that the emission spectrum of the donor must overlap the absorption spectrum of the acceptor. To satisfy the overlap criteria, the donor and acceptor that form the FRET pair are usually both fluorescent dyes. "Dark transfer" with the participation of a nonfluorescent acceptor is possible, but it often occurs via non-FRETbased mechanisms $(20,90,91)$.

$\kappa$ is the orientation factor describing the relative orientation of fluorophores. Two molecules participating in FRET are described by Förster's theory as coupled dipole oscillators. The probability of energy transfer is the highest when participating dipoles have parallel orientations but drops to zero when dipoles are perpendicular. Its value can theoretically be within the $0-4$ range. In case of random dipole orientation, the $\kappa$ value is estimated as $2 / 3$. This assumption is true for many but not all situations. In systems where the nonrandom orientation of dipoles is observed (e.g., biomembranes), the $\kappa$ value needs correction. Methods for $\kappa$ analysis in nonrandom orientations are presented in a number of specialized publications $(19,29,30,83)$. The $\mathbf{n}$ value indicates the refractive index of the medium between fluorophores. In an aqueous solution, its value is 1.33 .

The efficiency of energy transfer (E) 
is defined as the number of quanta transferred to the acceptor, divided by the number of quanta absorbed by the donor. It is described by the expression (26):

$$
E=\left(R_{0} / R\right)^{6 / 1}+\left(R_{0} / R\right)^{6}
$$

where $\mathbf{R}$ is the distance between the donor and acceptor (in $\AA$ ) and $\mathbf{R}_{\mathbf{0}}$ - distance (in $\AA$ ) at which energy transfer is $50 \%$ efficient, also called the Förster radius.

The formula shows that the rate of energy transfer is inversely proportional to the sixth power of the distance between the donor and acceptor. Therefore, the efficiency of the transfer rapidly declines to zero at distances larger than the Förster radius. The Förster radii have to be experimentally determined for each specific donor-acceptor pair, and the majority of fluorophore pairs fall within the $1-10 \mathrm{~nm}$ range. In other words, for FRET to occur, the donor and acceptor molecules must be within 1-10 nm of each other.

The specific limited distance at which energy transfer is effective forms a basis for utilization of FRET as a "molecular ruler", measuring 0.5-10$\mathrm{nm}$ distances within biomolecules with high precision $(74,86)$.

Oligonucleotides with DNA backbones provide a convenient moiety for the preparation of FRET-based probes because their properties are well known. However, many factors including base composition and temperature $(11,100)$, DNA topology $(11,98)$, and the number of chromophore tags (41) can complicate energy transfer in DNA constructs.

Interactions between the nucleotide bases and the fluorophore could be responsible for the quenching effect observed when ssDNA labeled with a single fluorescein is annealed to an unmodified complementary strand (44, 45). Using modified base 2-aminopurine (2AP) as an acceptor shows that energy transfer from adenine (A) is an order of magnitude higher than from other bases. Stacks of several As adjacent to 2AP act as antennae for 2AP, significantly increasing its excitation. Interposition of a $\mathrm{G}, \mathrm{C}$, or $\mathrm{T}$ base between $\mathrm{A}$ and the $2 \mathrm{AP}$ acceptor efficiently blocks the transfer from A to 2AP (100). Also, a rise in temperature can have a negative effect on the energy transfer efficiency (100), whereas interposition of the third chromophore acting as a relay station midway between the two chromophores significantly enhances it (41).

Parts of the fluorophore-labeled DNA probe can participate in collisional and static fluorescence quenching. These non-FRET-based mechanisms can mimic the fluorescence quenching effects of FRET $(20,43)$. On the other hand, the fluorescence suppression caused by collisional (dynamic) and static (complex formation) quenching $(20,43)$ is often used in its own right in DNA probes labeled with various dark quenchers that do not satisfy the essential spectral overlap criteria for FRET $(90,91)$. The dynamic quenching results from the collisional encounters between the quencher and the fluorophore so that, upon contact, the fluorophore returns to the non-excited ground state without the emission of a photon (43). In static quenching, a nonfluorescent complex is formed between the fluorophore and the quencher (43). In both cases, unlike FRET, the direct contact of the fluorophore and the quencher is required (20). Consequently, both dynamic and static quenching occur at distances shorter than those optimal for FRET; thus, both static and dynamic quenchers may be considered contact quenchers (20). Collisional quenching could involve an external, solute quencher that is not attached to the probe (e.g., the fluorophore on another probe) and an internal quencher attached to the probe $(20,90)$. Solute quenching reactions depend on diffusion and are inhibited by the high viscosity of solutions and, unlike FRET, increase with temperature (20). On the contrary, static quenching decreases with temperature, which is likely to destabilize the quencher-fluorophore complexes (43). In some instances, the fluorophore can be quenched by a com bination of collisions, complex formation, and resonance energy transfer with the same quencher $(20,43)$. In DNA probes with nonlinear configurations that constantly change their shape, the situation could include all of the above. For example, in the "breathing" (constantly opening and closing) hairpin constructs that have the donor and acceptor positioned on the opposing free DNA ends, both dyes could go through a wide range of distances, making it possible for all mechanisms of quenching to participate in the fluorescence suppression. The combination of FRET-based and contact quenching could also be present in other DNA probes that satisfy spectral overlap criteria when the donor and acceptor come in direct contact.

\section{DESIGNS AND APPLICATIONS OF DNA FRET PROBES}

Oligonucleotides with DNA backbones, labeled with one or several chromophores, can form FRET systems. They are used as FRET probes in a variety of reactions, signaling their occurrence via changes in the fluorescence energy transfer. FRET can occur when the duplex is formed between two labeled oligonucleotides, bringing the donor and acceptor dyes in close proximity. Alternatively, the hairpin configuration of the oligonucleotide dual-labeled with both donor and acceptor fluorophores can result in FRET. The detection of FRET and its disruption are both used in the assays. The detection of FRET implies that the two fluorophore molecules are physically within a few nanometers. FRET disruption indicates that the relative positions of the molecules have changed and the new distance between them is prohibitive to the occurrence of FRET.

DNA-based FRET probes are used in vitro and in vivo in a plethora of applications monitoring various types of DNA and RNA reactions, including PCR, hybridization, ligation, cleavage, recombination, and synthesis. They are also employed in sequencing, mutation detection, and as parts of biosensors to assess the concentrations of lead, DNA/RNA, and protein.

\section{DNA Hybridization Detection}

The hybridization reaction is one of the most important and sensitive tools in the molecular biology arsenal. Traditionally, hybridization has been performed as a heterogeneous assay using solid supports that allowed the subsequent separation of the duplexes formed between the probe and the target DNA from the unreacted probe 
(58). The use of solid supports was necessary to eliminate the obscuring background fluorescence of the unhybridized probe. The introduction of FRET-based hybridization probes improved hybridization technology in two important aspects: $(i)$ in FRET-based hybridization assays, the signal from the unreacted probes is quenched. This permits the real-time observation of the hybridization reaction directly in solution, as a homogenous type of assay, with no need for solid support and washing procedures. One advantageous feature of the homogenous assay is its speed and simplicity, allowing for the easy adaptation to automated performance. (ii) The high speed of hybridization in solution and the detection of only the hybridized probe signal makes it possible to perform in vivo hybridizations directly in live cells. Multiple formats of FRET hybridization probes have been developed (Figure 1).

Adjacent hybridization probes. Adjacent hybridization probes were introduced first. The assays utilized two oligonucleotide probes hybridizing to adjacent DNA sequences (Figure 1). The probes were end-labeled with chromophores so that one had a 3' donor and the other had a $5^{\prime}$ acceptor (32). The inverted position of a donoracceptor pair ( $3^{\prime}$ acceptor- $5^{\prime}$ donor) was equally successful (4). The FRET system is formed after hybridization, signaling the completion of the reaction by the quenching of the donor and the sensitization of the acceptor fluorescence. Adjacent probes of similar design were used to assess DNA conformation and to detect hairpins in stretches of ssDNA (64).

Measurements of the internucleotide distances in ss- and dsDNA chains were taken $(59,76)$, and it was demonstrated that, for the most frequently used fluorescein-rhodamine pair, the maximum transfer efficiency is obtained when the donor and acceptor are separated by four or five intervening nucleotides $(4,66)$. At shorter distances, the contribution of non-FRET-based contact quenching significantly increases (43). However, the new pyreneperylene donor-acceptor pair can participate in FRET at much shorter distances than the fluorescein-rhodamine pair and is advantageous in
FRET-based hybridization detection using the adjacent probe format (59).

An additional improvement in the design of adjacent hybridization probes was achieved by inserting two identical fluorophores close to each other into the donor probe. This modification significantly increases the fluorescence intensity of the acceptor fluorophore on the adjacent probe after hybridization (72). In contrast, inserting two fluorophores into the acceptor probe did not increase fluorescence, probably because of self-quenching interactions. The BODIPY ${ }^{\circledR}$ 650/665 fluorophores showed the highest efficiency in the enhancement of FRET, whereas Alexa ${ }^{\mathrm{TM}}$ 488 had no effect. This signal amplification by means of the double-labeled donor probe could be applied to the other FRET hybridization probes (72).

A recent clinical application of adjacent fluorescent probes resulted in the amplification and fluorescent genotyping of the cystic fibrosis F508del locus from human genomic DNA in less than $30 \mathrm{~min}$ (27). The hybridization of the probes was monitored by the changes in energy transfer between fluorescein and Cy5 during heating and cooling.

Molecular beacons. One of the latest developments in the area of hybridizing DNA FRET probes was the introduction of molecular beacons (89). Paradoxically, it is not FRET per se but its disruption that is primarily used in these probes. The FRET phenomenon is used as a "molecular cloaking device", hiding the probe's fluorescence until it attaches to its target hybridization sequence. Molecular beacons are designed as hairpin-shaped oligonucleotides (Figure 1). The stem is formed by the terminal $5-8$ bases, while the central single-stranded portion assumes a loop configuration. It can be 15,25 , or 35 nucleotides long (89). The doublestranded stem carries the donor and acceptor dyes. The loop fragment can hybridize to the target nucleic acid sequence. The rationale of this approach is that, when the probe is not hybridized to the target, the donor and acceptor are brought in close proximity by the stem formation, resulting in the FRET-based quenching of the donor. However, in the presence of the target sequence, the probe forms longer and stronger hybrids with the target than by forming the stem. The FRET transfer is disrupted, thus resulting in the appearance of the donor fluorescence. The fluorescent acceptor fluorophore in molecular beacons is replaced with the nonfluorescent quencher, DABCYL [4(4'-dimethylaminophenylazo) benzoic acid]. The advantage is that the probe detection is simplified because it does not fluoresce in a nonhybridized form. Further analysis of DABCYL-based quenching revealed that its mechanism is not FRET-related (90). Quenching was observed with various fluorophores, with no need for spectral overlap with the quencher molecule. How ever, because other quenchers are possible, we will discuss these probes.

There is evidence that the molecular beacon probe design can be improved. When both the fluorophore and quencher were positioned on the same short peptide sequence, 200-fold quenching was observed. However, in molecular beacons, only a 25 -fold increase in fluorescence is detected after hybridization (89).

The molecular beacons proved useful in situations in which it is inconvenient to isolate probe-target hybrids, such as the detection of targets within living cells $(61,84)$. They found additional applications as biosensors (51, 52) to measure the cleavage of ssDNA (48), to detect protein-nucleic acid interactions (49), and in genotyping alleles of the human gene (42). We will discuss some of these applications later in this review.

Multicolor molecular beacons have been designed recently for the simultaneous detection of multiple targets (90). The multicolor molecular beacons are advantageous in multiplex PCRs; how ever, their different reporter fluorophores cannot be optimally excited by the same monochromatic light source employed in many devices that monitor fluorescence. The recently introduced wavelength-shifting molecular beacons overcome this limitation (91).

Wavelength-shifting molecular beacons contain three labels instead of two. They do not form the blunt-ended hairpin but instead contain a $5^{\prime}$ extension with the emitter fluorophore positioned at the $5^{\prime}$ terminal. The additional "harvester" fluorophore is positioned internally some distance from the emitter so 
that it is located directly opposite from the 3' quencher in the hairpin formation. This probe design is based on the hypothesis that energy transfer from the harvester to the quencher is faster than the transfer to the emitter (91). When probes are not hybridized to the target, they assume a hairpin formation and the transfer of energy from the harvester to the emitter is blocked by the quencher. When the hairpin conformation is disrupted after hybridization, the harvester fluorophore transfers energy to the emitter, boosting its fluorescence.

Wavelength-shifting molecular beacons utilize non-FRET-based quenching and the resonance energy transfer. In the hairpin conformation, the fluorophore and the opposing nonfluorescent quencher are so close that they form a transient nonfluorescent complex that dissipates light energy as heat by the non-FRET-based mechanism. However, when the probe hybridizes to its target and the quenching complex is disrupted, the energy from the first, previously quenched fluorophore (the harvester) is transmitted to the second (the emitter) fluorophore via FRET.

Wavelength-shifting molecular beacons combine principles used in the classical molecular beacon design with the advantages of the energy transfer sequencing primers (described later in this review). They are not only significantly brighter than the conventional molecular beacons but can also emit fluorescent light at different wavelengths. After being excited by a monochromatic light source, their uniform harvester fluorophore can transmit energy to different emitter fluorophores (91).

These probes were used to simultaneously detect two different single-nucleotide polymorphisms (91). It was proposed that the combination of these probes with conventional molecular beacons would enable the reliable detection of a much higher number of targets.

Double-stranded hybridization probes. Another approach uses a double-stranded probe formed by two com plementary oligonucleotides. One of the oligonucleotides is $5^{\prime}$ end-labeled with the donor fluorophore, and the other has a 3 ' acceptor. The rationale of the assay is to detect the target sequence by com petitive hybridization of one of the oligonucleotides forming the probe du- plex. When the initial probe duplex is disrupted, because of the hybridization of one of the oligonucleotides to the target sequence, the quenching of the donor is stopped and its fluorescence becomes observable. In a demonstration of the practical utility of the assay, fluorescein was used as a donor and either pyrenebutyrate or sulforhodamine 101 was used as an acceptor (65). The assay detected as little as $2 \times 10 \% \mathrm{~mL}$ of the target DNA sequences. The use of FRET probes of similar design allowed for the detection of hybridization events occurring in live cells (81).

Hybridization probes in combination with an intercalator. Another approach utilizes a hybridization probe labeled with only an acceptor fluorophore. It works in combination with a low molecular weight donor dye, which intercalates into the target DNA. When the probe hybridizes to the target sequence, the energy transfer occurs from the intercalator to the acceptor, increasing its fluorescence. The approach was successfully tested with the intercalating dye acridine orange serving as a donor and rhodamine at the $5^{\prime}$ end of the oligonucleotide probe acting as an acceptor (4).

Recent developments in the field of FRET-based hybridization detection include a method for the monitoring of oligonucleotide hybridization on microparticles (33) and the introduction of a new pyrene-perylene donor-acceptor pair for hybridization assays (59).

The particular advantage of the pyrene-perylene donor-acceptor pair is in its high quantum efficiency, permitting the higher sensitivity of measurements. An additional advantage is its suitability for the measurements of much shorter Förster distances (11-32 $\AA$ ) in nucleic acid probing, compared to the regularly used fluorescein-rhodamine pair (25-75 ̊) (59).

\section{PCR Monitoring}

The energy transfer probes used to monitor DNA amplification in PCR are either cleaved in the reaction (as TaqMan ${ }^{\circledR}$ probes), incorporated into amplified DNA (as Scorpion primers), or undergo a conformation change in the presence of a complementary DNA target (as molecular beacons). Many probe designs employ oligonucleotides 
that form reversible stem-loop configurations. In each case, the probes signal the reaction occurrence by eliminating the quenching influence on the donor fluorophore.

$5^{\prime}$ nuclease assay for PCR monitoring (TaqMan probes). The $5^{\prime} \mathrm{nu}$ clease assay was developed to allow the real-time monitoring of the reaction. In its initial format, it did not use FRET but relied on radioactive labeling instead (35). FRET-based detection was added later $(53,54)$. The assay utilizes the $5^{\prime} \rightarrow 3^{\prime}$ exonuclease activity of Taq DNA polymerase (Applied Biosystems, Foster City, CA, USA) to monitor the ongoing reaction. Taq DNA polymerase can cleave $5^{\prime}$ terminal nucleotides from the strand of DNA that it displaces while synthesizing a new strand (56). The cleavage primarily occurs at the junction between the displaced singlestranded portion and the double-stranded paired part of the DNA strand. This results in the release of mono- and oligonucleotides from the $5^{\prime}$ end of the displaced DNA strand. That property of Taq DNA polymerase was used to monitor the reaction (Figure 2). The FRET DNA probes in the $5^{\prime}$ nuclease assay

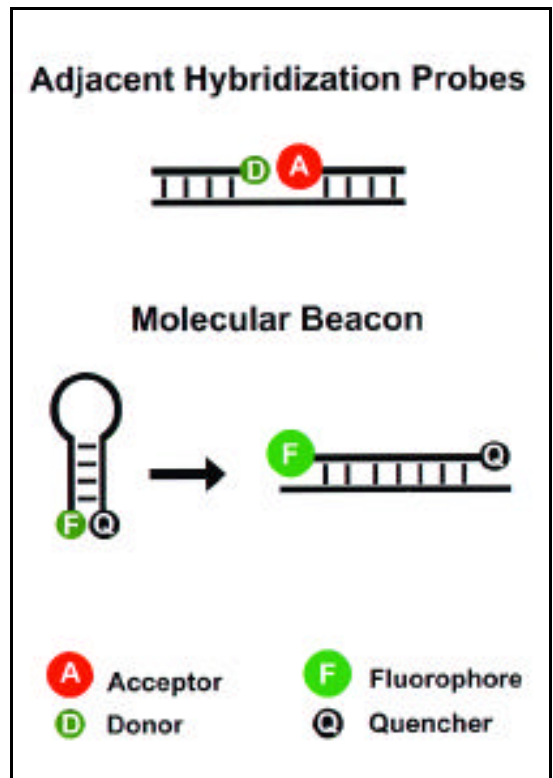

Figure 1. Designs of FRET probes for hybridization detection. Adjacent hybridization probes. The fluorescence of the acceptor fluorophore is enhanced when two probes hybridize to the target sequence. Molecular beacons. Successful probe hybridization changes its configuration. The separation of the fluorophore from the quencher restores fluorescence. are short oligonucleotides, complementary to the target sequence in the amplified DNA and 3 '-modified so that they cannot be extended on the $3^{\prime}$ end. They are labeled with fluorophores at both the $3^{\prime}$ and $5^{\prime}$ ends. The reporter dye is positioned at the $5^{\prime}$ end, and the quencher is positioned on the $3^{\prime}$ end of the probe. With the probe intact, the reporter dye fluorescence is quenched. In the reaction, the probe hybridizes to the target sequence. During PCR, it is cleaved by the $5^{\prime}$ nuclease activity of Taq DNA polymerase. The cleavage separates the quencher from the reporter and restores its fluorescence.

In an early work (46), probes of sim ilar design successfully discriminated between wild-type and mutated sequences in the human cystic fibrosis gene by nick-translation PCR. Two probes were used, one for the normal sequence and the other complementary to the sequence containing a 3-bp deletion. Each probe had a different reporter fluorophor at the $5^{\prime}$ end and a common quencher dye attached to the seventh nucleotide from the $5^{\prime}$ end. The identity of the target DNA was determined from the fluorescence emission spectrum.

A cost-effective method to prepare and evaluate double-labeled probes for the $5^{\prime}$ nuclease assay is described in Reference 77. The guidelines for the probe design are discussed in Reference 54.

Molecular beacons. Strictly speaking, the fluorescence quenching in molecular beacons that use DABCYL is not FRET-related because it does not

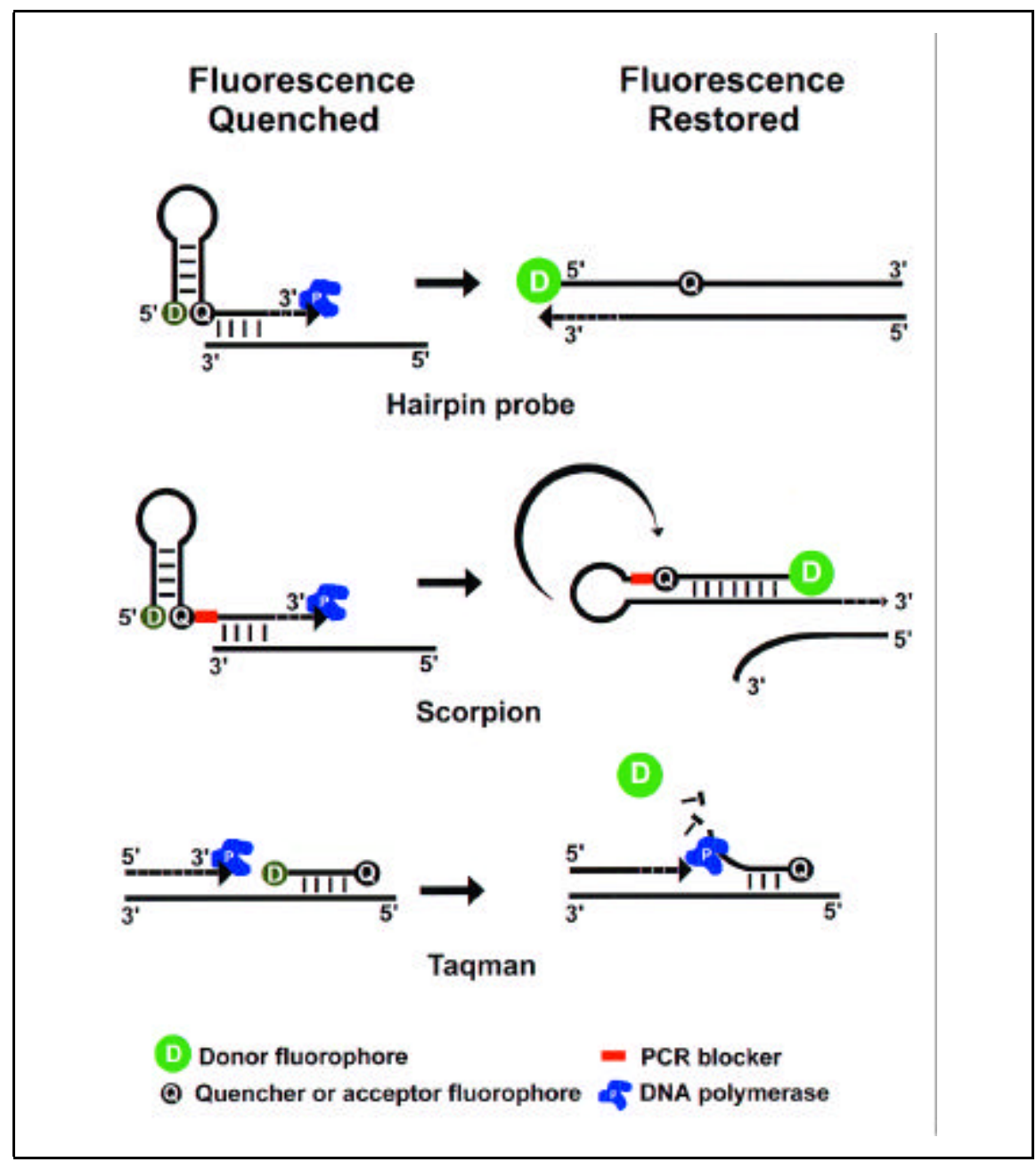

Figure 2. Designs of FRET probes for PCR monitoring. Hairpin probes. They signal ongoing PCR after the hairpin is disrupted by the newly synthesized DNA strand. Scorpion primers. They change configuration and self-hybridize after the primer is extended in PCR. TaqMan probes. The probes are destroyed by Taq DNA polymerase in the course of PCR, separating the donor and acceptor fluorophores. 
satisfy the spectral overlap criteria (90). However, the FRET-based quenching can also be used.

Molecular beacons found applications in PCR soon after their introduction as hybridization probes $(73,75)$. They can monitor an ongoing reaction and discriminate alleles in real-time PCR assays using the same mechanism - by hybridizing to their complementary DNA targets. The products could be detected by adding the finished reaction to a microtiter well containing immobilized molecular beacon probes and by reading the generated fluorescence (73). Alternatively, the closed-tube, real-time format can be used $(75,94)$. In this case, molecular beacons are added directly to the PCR mixture and hybridize to the newly synthesized DNA in the course of the am plification reaction. Compared to TaqMan probes, molecular beacons are advantageous in multiplex PCR assays because, in theory, they can determine more products simultaneously (94).

Scorpion primers. The Scorpion primers also take advantage of the stem-loop configuration. They consist of a hairpin linked to the $5^{\prime}$ end of a PCR primer via a "PCR stopper" that prevents polymerase read-through. The loop is complementary to a sequence in the strand, which is newly synthesized in a reaction as a continuation of the primer. The donor fluorophore is attached to the $5^{\prime}$ end of the stem, and the quencher moiety (normally methyl red) is positioned $3^{\prime}$ of the donor just after the loop sequence so that, after the stem formation, the quencher is located opposite of the donor (Figure 2) $(88,97)$. When added to the reaction, the whole Scorpion primer is incorporated into the PCR product. During the cooling step of the reaction, the Scorpion primer folds back and hybridizes to the complementary sequence in the newly synthesized part of the same DNA strand. This eliminates the quenching of the donor fluorophore and signals a successful amplification $(88,97)$. The hybridization reaction occurs within the same strand and is faster than the intermolecular hybridizations of molecular beacon probes. A comparative study of Scorpions, TaqMan, and molecular beacons using a LightCycler ${ }^{\circledR}$ (Roche Molecular Biochemicals, Indi- anapolis, IN, USA), showed the advantage of Scorpion primers, especially in fast cycling conditions. These primers can be valuable tools in mutation screening and are easily adaptable for high-throughput analysis (88).

An interesting earlier approach uses the advantages of the hairpin configuration in probe design in a manner different from that of Scorpion primers (70). In this approach, a molecular beacontype probe is attached to a regular PCR primer. In the course of the reaction, the probe is incorporated into the product where it looses the stem-loop configuration and becomes linear. This separates the fluorophor from the quencher, signaling a successful amplification (Figure 2). Because the donor and acceptor are kept close in a hairpin, the probe has less background fluorescence compared to TaqMan probes, in which the donor and acceptor are positioned at relatively longer distances on the opposite ends of the linear DNA probe. The assay allows real-time quantitation of the DNA amplification reaction.

The principal difference among various probes for PCR monitoring is that the probe is either incorporated or not incorporated into the final product. The molecular beacons and TaqMan probes, unlike the Scorpion and hairpin primers, are not incorporated into the final PCR product. This gives them an additional advantage because they can target and recognize any specific sequence within a product. In other words, unlike in other probe designs, they are not limited to the detection of the sequences present in close proximity to the incorporated primer (as in Scorpion or hairpin primers) and can detect any particular product directly. Thus, several different probes for the same product can be used to increase the reliability of detection. This accounts for the ability of these probes to reliably measure amplified products even in the presence of amplification artifacts.

In addition to the assays described above, other PCR-based techniques with FRET as the reporter method were used for the rapid detection of the factor V Leiden and hemochromatosis mutations (71), for the detection and quantitation of squamous cell carcinoma antigen 1 and 2 mRNAs (67), and for the genotyping of single-nucleotide 
polymorphisms $(5,7)$. The advantages of FRET as a reporter were also utilized in signal amplification in isothermal conditions (31) and in commercially available PCR machines that use FRET to monitor amplification reactions (14, $55,68,69,99)$. However, the extensive research using thermal cyclers with FRET capabilities and the numerous applications developed in this area are beyond the scope of this review and deserve a special discussion.

\section{DNA Mutation Detection}

The oligonucleotide FRET probes developed for the detection of mutations can employ either the disruption (Invader probes) or creation [templatedirected dye-terminator incorporation assay (TDI assay)] of energy transfer systems.

Similar to the $5^{\prime}$ nuclease assay (TaqMan probes), the Invader assay employs degradable probes; however, it uses not one but two degradable (and one nondegradable) probes working in concert. Both the hairpin-shaped and linear oligonucleotides are used to create branched DNA structures, which are subsequently destroyed in the reaction. The other unique feature of this technique is that it incorporates efficient signal amplification and, thus, does not require PCR to detect very small amounts of its target sequences.

The distinctive feature of the TDI assay is that FRET probes are synthesized in the process of detection. How ever, the assay requires the PCR amplification of its target DNA before the detection step.

Invader assay. The Invader assay was introduced for the detection of mutations in complex mixtures of nucleic acids and the quantitation of target DNAs. In its original format, it did not use FRET and had relatively low sensi- tivity (57). Its later modification, the serial invasive signal amplification reaction, employs FRET and can detect less than 1000 target molecules with no prior target amplification (31). Good concordance between allele-specific PCR and the Invader assay was reported in a study of the factor V Leiden mutation (34).

The assay uses a unique combination of three oligonucleotide probes (two linear and one hairpin-shaped) (31) and structure-specific $5^{\prime}$ nucleases (Cleavases $\left.{ }^{\circledR}\right)$ that cleave single-stranded, partially hybridized DNA (50). The reaction proceeds in two steps. In the first step, two linear, partially overlapping oligonucleotides, called the Invader and the signal probe (or the upstream oligonucleotide and the probe), hybridize to the target DNA. The Invader probe overlaps the signal probe by one nucleotide. The signal probe contains two regions, a part complementary to the target DNA and a 10-base-long 
noncomplementary $5^{\prime}$ arm region, which form a single-stranded flap after hybridization. The flap consists of the noncomplementary $5^{\prime}$ region of the signal probe plus a single complementary nucleotide that was displaced by the Invader probe. This branched structure serves as substrate for the Cleavase and is cut off in the reaction.

In the second step, the cleaved $5^{\prime}$ arm hybridizes to the hairpin-shaped FRET probe. This probe contains a 10base-long 3' overhang, complementary to the cleaved $5^{\prime}$ arm. In addition, the hairpin probe contains fluorescein and Cy-3 fluorophores, which form a FRET pair. Fluorescein is attached to the terminal $5^{\prime}$ end of the hairpin, and $\mathrm{Cy}-3$ is several nucleotides farther up the stem. The 11-nucleotides-long cleaved 5' arm hybridizes to the 10-base-long overhang of the hairpin, and its eleventh nucleotide displaces the terminal 5' nucleotide of the hairpin labeled with fluorescein. The unhybridized nucleotide is subsequently cut off by the Cleavase, disrupting the FRET system and signaling the detection. The reaction occurs at a temperature close to the melting point of the hybrid, which allows for the turnover of the signal probe without temperature cycling (31).

The assay can quantitate target DNAs and RNAs down to subattomolar concentrations and is able to discriminate single-base differences in the sequence of its targets (31). It was shown to be a useful tool in mutation research and for large-scale genotyping of single-nucleotide polymorphisms $(63,80)$.

Template-directed primer extension. A TDI assay is a simple technique developed for the detection of single base pair changes in DNA $(6,8)$. As a probe, it utilizes a single linear primer that is $5^{\prime}$-labeled with fluorescein. The reaction starts with total human DNA, and the sample is amplified by PCR. This is followed by the enzymatic degradation of excess primers and dNTPs using exonuclease I and alkaline phosphatase. After that, the primer extension reaction is performed. In the reaction, the amplified DNA fragments are at first incubated with a fluoresceinlabeled probe designed to hybridize to the DNA template adjacent to the mutation site. Primer extension occurs in the presence of a modified Taq DNA poly- merase (Klentaq1-FY) and two allelic terminators-ddNTPs labeled with either 6-carboxy-X-rhodamine (ROX) or $\mathrm{N}, \mathrm{N}, \mathrm{N}, \mathrm{N}$,-tetramethyl-6-carboxyrhodamine (TAMRA) dyes. In the mutation site, the template contains the base complementary to either ROX- or TAMRA-labeled ddNTP. Thus, depending on the presence or absence of the mutation, one dye but not the other is added to the fluorescein-tagged probe, creating the FRET system. The mutational status of DNA is determined by analyzing the real-time fluorescence intensity profiles of the three dyes in the reaction mixture using a fluorescence thermal cycler (8). This simple and easily standardized assay could be quickly adapted for high-throughput genotyping (8).

\section{DNA Cleavage Detection}

The $5^{\prime}$ nuclease assay discussed above (see PCR Monitoring) signals the PCR amplification by actually detecting the cleavage of DNA-based TaqMan probes. However, it is not the sole example of the application of FRET for the visualization of nuclease activity. DNA cleavage is a logical target for FRET-based assays because the separation of the donor and acceptor fluorophores positioned on a DNA probe can be easily observed. The probes used in DNA cleavage detection are either linear or hairpin-shaped, dual-labeled oligonucleotides. Multiple assays were developed to report the activity of various nucleases.

Monitoring the kinetics of the PaeR7 endonuclease activity was demonstrated in an assay that uses a series of double-stranded probes containing the recognition site for PaeR7 (CTCGAG). The probes were dual-labeled with fluorescein and rhodamine, as the donor and acceptor, with a 6-mer spacer between them. The activity of the endonuclease was detected as an increase

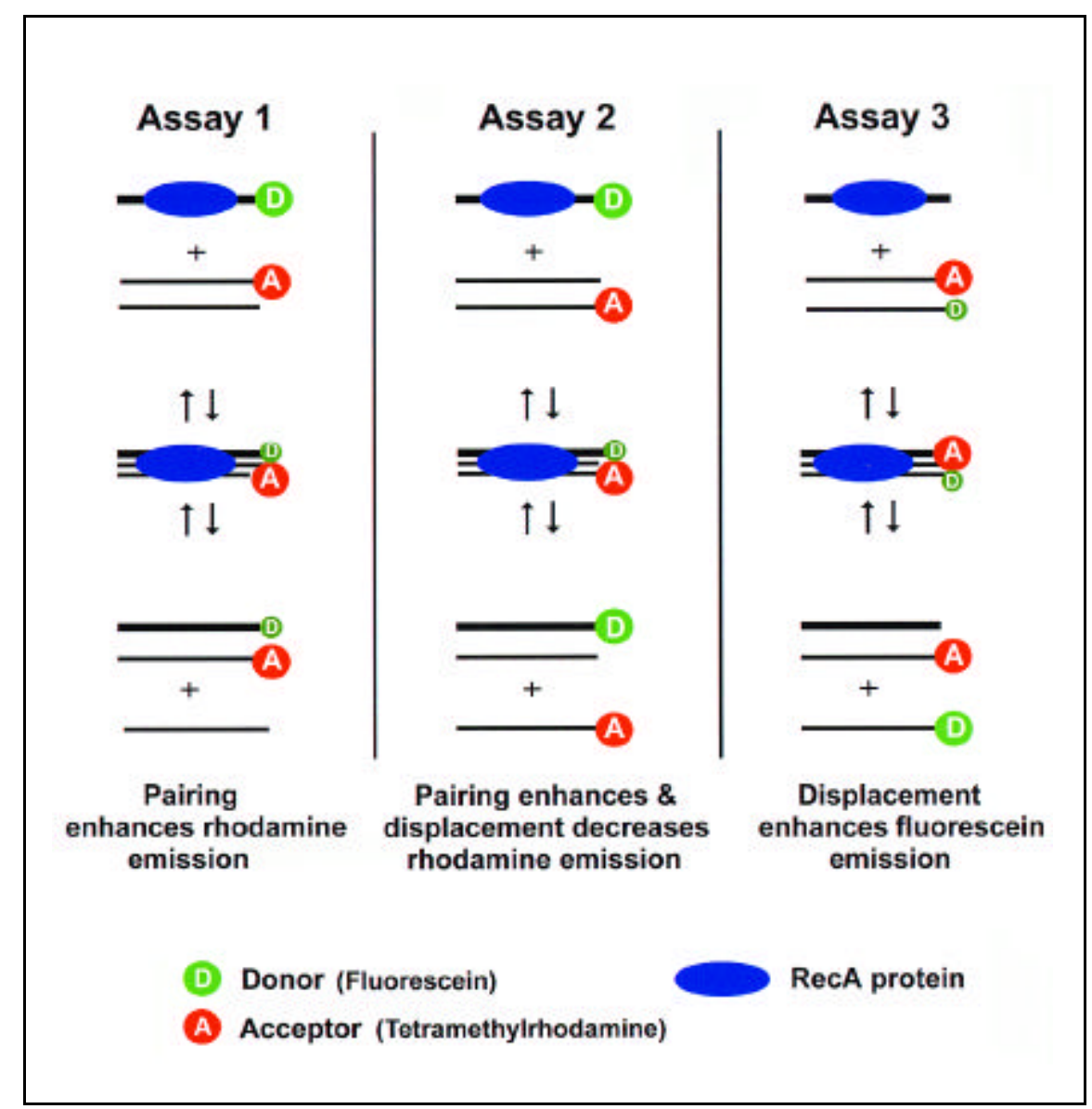

Figure 3. Three FRET assays for the detection of different stages in DNA recombination. 
of the donor fluorescence (25).

Another assay was introduced to measure the integrity of oligonucleotides in solution or in live cells after transfection (92). It uses a dual-labeled, 10-mer oligonucleotide with fluorescein positioned on the $3^{\prime}$ end and rhodamine- $X$ on the $5^{\prime}$ end. For in vivo testing, the oligonucleotide was injected into sea urchin eggs and was then excited by blue light (470-490 nm). Green (520-560 nm) and red (greater than $580 \mathrm{~nm}$ ) images were taken at regular intervals after injection using a fluorescent microscope. The red/green ratio decreased with time, signifying the disruption of resonance energy transfer caused by the cleavage of the oligonucleotide. The technique can be useful for in vitro and in vivo studies of nucleolytic activity and for assessing the integrity of transfected nuclease-sensitive oligonucleotides.

The application of FRET for the detection of endonucleolytic activity of HIV-1 integrase was described in Reference 45 . Integrase protein cleaves a specific DNA sequence within long terminal repeats of viral DNA. The probes mimicked the substrate of the integrase and consisted of a duplex with a long $3^{\prime}$ overhang formed by 49- and 31-mer oligonucleotides. Nineteen base pairs in the duplex corresponded to the sequence at the U5 end of HIV-1 DNA, preceded by nine random base pairs at the $5^{\prime}$ end to aid in annealing. The strands of the duplex were labeled with fluorescein and eosin, forming a donoracceptor pair. The cleavage results in the separation of the fluorescein isothiocyanate (FITC)-labeled overhang of the probe from the eosin-labeled section, which is followed by the enhancement of FITC fluorescence intensity. The assay was proposed for the rapid screening of novel integrase inhibitors.

The advantages of the hairpin-probe configuration for the detection of DNA cleavage were explored in the assay, which used the molecular beaconbased probes to visualize the activity of single-strand specific nucleases (48). The method monitored the reactions of DNase I, Mung Bean nuclease, and S1 nuclease. The probes consisted of two parts, the single-stranded loop and the double-stranded stem, end-labeled with donor and acceptor dyes. When a nu- clease cleaves the single-stranded loop portion of the probe, the stem oligonucleotides separate because the melting temperature of the duplex is significantly less than the temperature of the cleavage reaction $\left(37^{\circ} \mathrm{C}\right)$. Separation of the stem disrupts energy transfer between the donor and acceptor-quencher and restores the donor fluorescence.

The popular hairpin configuration was also used in the new "molecular break light" probes. They were introduced for the continuous monitoring of DNA cutting by enediynes. The enediynes are small molecules with DNAcleaving capabilities and are promising anticancer drug candidates (3). In addition to enediynes, the assay assessed the other DNA cleaving agents, such as bleomycin, iron-EDTA, and the restriction endonuclease $\mathrm{Ba} \mathrm{HI}$.

These new probes possess features of both molecular beacons and TaqMan probes. Structurally, they resemble molecular beacons, but their mode of action is similar to TaqMan probes. Molecular break lights are hairpinshaped oligonucleotides with a fluorophore donor on the $5^{\prime}$ end and an acceptor-quencher on the $3^{\prime}$ end. The stem of the probe contains a DNA sequence susceptible to cleavage by a specific DNA-cleaving agent. When the stem is cleaved by a specific agent, the fluorophore-quencher pair separates and the fluorescence of the donor dye becomes observable, signaling the occurrence of the reaction. The probe is destroyed in the reaction, thus resem bling the TaqMan-degradable probe in its mode of operation. In the 10-bp stem of the hairpin, the break light probes contained either a $5^{\prime}$-TCCT-3' sequence, recognizable by a representative of the enediyne family such as calicheamicin, or a 5'-GGATCC-3' BamHI recognition sequence. Similar to molecular beacons, the $5^{\prime}$ fluorophore in the break light probes was fluorescein-based (FAM) and the 3' quencher was DABCYL (3). The advantage of the assay is in its high signal-to-noise ratio (approximately 40) and its ability to continuously monitor the ongoing reaction.

\section{DNA Ligation Detection}

FRET DNA probes have been used to signal successful ligation inside live cells (15) as a part of ligation-based apoptosis detection (16-18). The probes consist of two complementary 15-mer oligonucleotides end-labeled with FITC and rhodamine. When the strands are combined to form a duplex, the fluorophores positioned on opposite strands form a donor-acceptor pair. The

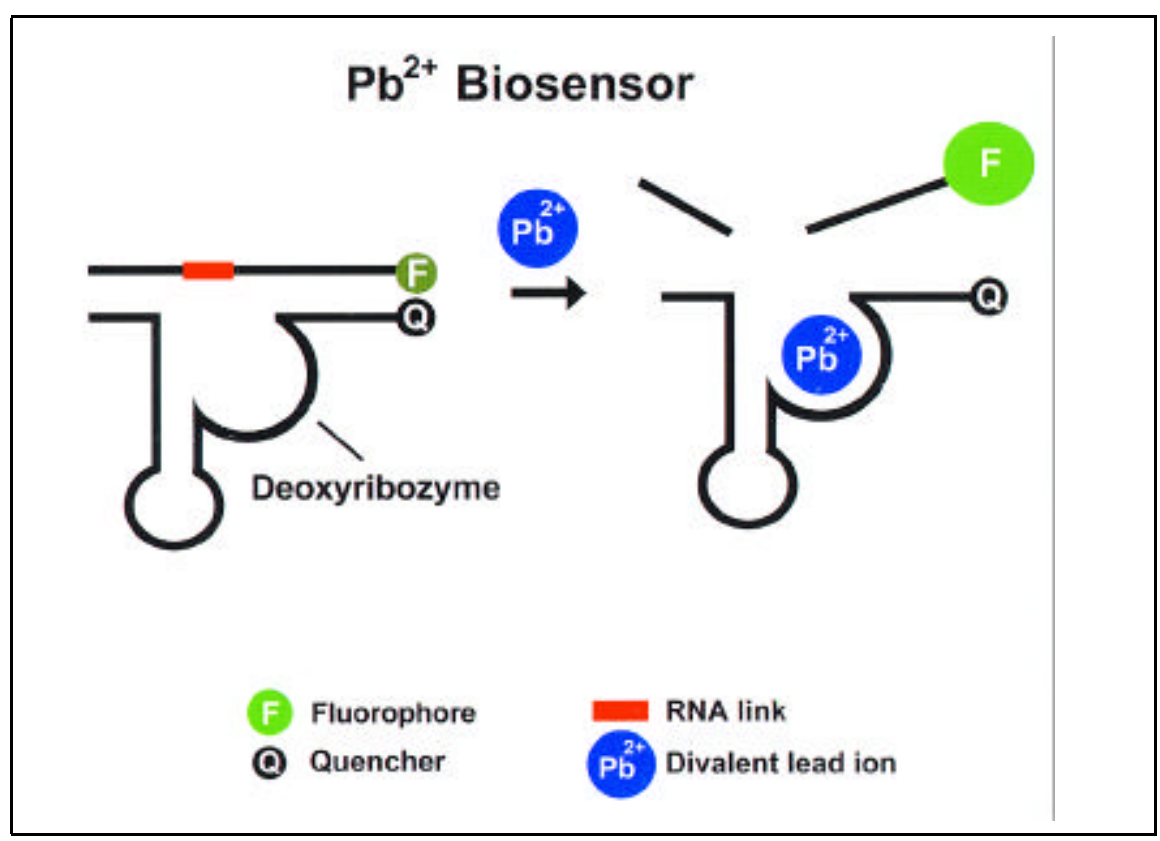

Figure 4. Deoxyribozyme biosensor. It is activated by lead ions and reports their presence by fluorescence enhancement. 
ligatable end of the probe has terminal $3^{\prime} \mathrm{OH} / 5^{\prime} \mathrm{OH}$. DNA ligase attaches to only the $3^{\prime} \mathrm{OH}$ strand bearing the FITC, whereas the rhodamine-labeled $5^{\prime} \mathrm{OH}$ strand remains unligated and can be displaced by the subsequent addition of dNTPs and Klenow polymerase. This enhances FITC fluorescence. The probes combined with ligase were delivered into apoptotic cells using polyethyleneimine and ligated to the ends of the apoptotic DNA. The ligation was visualized when the cells were fixed and treated with polymerase.

\section{DNA Recombination Detection}

The FRET-based measurements of DNA recombination catalyzed by $E$. coli RecA protein (1) used the set of three 83-mer oligonucleotides. By varying the placement of the fluorescent tags attached to the oligonucleotides, DNA pairing and strand displacement steps in DNA recombination were assessed. Three assays to measure different steps in DNA recombination were described. The first two detect DNA pairing, and the third detects strand displacement reactions. (Figure 3).

In the first pairing assay, the RecA carrying 83-mer with $3^{\prime}$ fluorescein reacts with a duplex formed by two other 83-mers (one rhodamine-labeled), displacing the unlabeled strand and pairing with the rhodamine-labeled strand. As a result, fluorescein emission is quenched and rhodamine is enhanced.

For the second pairing assay, the same RecA carrying 83-mer with 3' fluorescein reacts with a duplex. However, this time the other strand in the duplex is rhodamine-labeled. The reaction results in the displacement of the rhodamine-labeled strand. The FRET system is formed during this pairing step and is disrupted after strand displacement. Rhodamine emission is enhanced only when three strands are in close proximity and then decreases again after displacement. This detects both the pairing and displacement phases.

The third (displacement) assay uses an unlabeled RecA-carrying, 83-mer that reacts with the duplex, where both strands are end-labeled with fluorescein and rhodamine, which form the FRET system. Strand displacement results in the enhancement of fluorescein and the decrease of rhodamine emission. The combination of results from the three assays provides information about all phases of the reaction. Similar approaches were used to study the other proteins of the RecA family (28).

\section{RNA Synthesis Monitoring}

Real-time monitoring of RNA synthesis in transcription reactions in vitro was performed using adjacent hybridization probes similar to those in 
DNA hybridization studies (78). Two 15-mer oligonucleotides were labeled with BODIPY 493/503 (acceptor) and Cy5 (donor). Excess amounts of the donor and acceptor DNA probes were used to visualize the progression of the RNA synthesis. Energy transfer occurred between fluorophores when the probes hybridized to adjacent regions on the newly synthesized RNA strand. The reaction was monitored by the changes of the fluorescence spectra. It was concluded that the probes hybridized to the newly synthesized RNA molecules before they folded to form secondary structure. This eliminates the need to select specific sites on the RNA for the probes because RNA folding does not prevent successful hybridization.

\section{Energy Transfer Sequencing Primers}

Energy transfer primers (ET primers) were developed to solve the problems with conventional single fluorophore primers used in the Sanger dideoxy chain-termination sequencing method. This method is currently the major sequencing approach and is widely used in automatic sequencing. In this approach, the four bases are identified by four distinct emissions in the sequencing reaction (82). Ideally, the four colored primers used in sequencing require three criteria (26). Each of the four fluorescent dyes should (i) have strong absorption at the wavelength common to three other fluorescent dyes used in sequencing, (ii) have emission maximum at a wavelength distinctly different from the other dyes, and (iii) initiate the same electrolytic mobility shift of DNA fragments.

Traditionally used single fluorophore primers do not satisfy $(i)$ and (iii), causing problems with automatic versions of the method. The rationale for the introduction of ET primers was the idea of a "composition fluorophore" in which the emission and absorption properties can be independently tuned using FRET. The goal was to enhance the emission of the acceptor by transferring to it additional energy from the donor. The use of the same donor fluorophore and the various acceptors of similar sizes would result in similar electrophoretic mobilities of the primers (26).
The original ET primers were introduced in 1995 (38). They carried 6-carboxyfluorescein (FAM) at the $5^{\prime}$ end as a universal donor and different acceptors at the $3^{\prime}$ end. The acceptors were attached to the modified thymidine residue and included 2', $7^{\prime}$-dimethoxy-4',5'-dichloro-6-carboxyfluorescein (JOE), ROX, or TAMRA. The optimal distance between the donor and acceptor for the energy transfer was determined to be between 6 and 10 nucleotides. With common excitation at $488 \mathrm{~nm}$, the emission of the primers was 1.4-24 times stronger when compared to single dye primers labeled with only the acceptor fluorophore $(36,38,40)$.

The system was subsequently improved in two regards. At first, the common fluorescein-based donor (FAM) supplying fluorescent energy to the acceptor fluorophores was replaced by a novel cyanine-based dye 3-(epsilon-carboxypentyl)-3-ethyl-5,5'-dimethyloxacarbocyanine (CYA) (37). The new primers had perfect mobility match, and their fluorescence intensity was 0.8 to 1.7 -fold higher than corresponding FAM-carrying ET primers (37). Most importantly, they displayed much lower levels of cross-talk among the four detection channels caused by the low fluorescence quantum yield of the CYA donor fluorophore.

The next improvement was the introduction of the "universal ET cassette" (39). The cassette can be added to the $5^{\prime}$ end of any primer.

Outside of the field of DNA sequencing, the ET primers found application in the visualization of the electrophoretic separation of DNA fragments. They allow single-base resolution for ds- and ssDNA fragments $(95,96)$. They proved to be convenient tools in this regard because of the large Stokes shifts of different primers, allowing excellent separation of two fluorescent signals. These advantageous features of the primers were used in the multicolor sizing of human short tandem repeat regions (95). Recently, ET primers were used in the high-throughput assay for the detection of microsatellite instability and the loss of heterozygosity associated with primary cancers (62). ET primers were also used for the detection of telomerase activity in the closed-tube-format telo- meric repeat amplification protocol (TRAP) assay. The system eliminates the need for post-PCR processing, reducing the risk of carryover contamination (93). The basic principle for ET primers of the composition fluorophore is also employed in wavelength-shifting molecular beacons (91).

\section{DNA-Based Biosensors Utilizing FRET}

The field of biosensors using FRET is new. These systems can combine several reactions for the detection of specific analytes. DNA-based biosensors utilize probes that are more structurally complex than those used in other applications. Indeed, out of the three examples discussed here, two contain deoxyribozyme sequences and the third one is the fiberoptic surface-immobilizable variation of molecular beacons.

A new analytical biosensor uses deoxyribozyme-based FRET probes in the determination of lead ions (47) (Figure 4). The research conducted at the University of Illinois, Urbana-Champaign identified a DNA oligonucleotide that cleaves substrate RNA in the presence of a lead cofactor. In the assay, they labeled the catalytic DNA with a fluorescent quencher, whereas the RNA substrate, partially complementary to the DNA oligonucleotide, was labeled with a fluorescent tag. In a duplex, fluorescent signal was suppressed because of the close proximity of the quencher to the fluorophore. However, if lead cofactor is present, then the cleavage occurs, releasing fluorescent tag into solution, which results in FRET disruption and the upregulation of the fluorescent signal. The sensor demonstrated an 80-fold selectivity for lead over other divalent metal ions. The approach described could result in the development of different assays capable of detecting a wide range of various analytes.

Another biosensor utilizing the ribonuclease activity of a deoxyribozyme and FRET as a reporter method was introduced to assess the concentrations of protein effectors through a change in a fluorescence signal (85). The approach uses a previously reported deoxyribozyme capable of cleaving a chimeric oligonucleotide substrate containing a single embedded ribonucleotide. When 
the substrate is $5^{\prime}$-labeled with a donor (fluorescein) and 3'-labeled with an acceptor (tetramethylrhodamine), its cleavage can be easily detected by the increase in donor fluorescence. The deoxyribozyme was modified by strategically placing a single biotin close to the loop region so that its binding with streptavidin prevents the appropriate folding of the deoxyribozyme and disrupts the substrate cleavage. The assay was able to reliably detect streptavidin at concentrations less than $1 \mathrm{nM}$ after an extended incubation time (approximately 1 day). The approach can be modified to assess concentrations of the other ligands.

A fiberoptic DNA biosensor uses modified molecular beacon probes to detect its target DNA molecules in real time (52). It employs a surface-immobilizable variation of the molecular beacons, which are biotinylated and have tetramethylrhodamine as the fluorophore and DABCYL as the quencher (21). These beacons were immobilized onto an optical fiber surface with the help of biotin-avidin interactions. The detection of their target sequence restores the tetramethylrhodamine fluorescence, which is recorded by a charge-coupled device (CCD) camera attached to the fluorescent microscope. The earlier version of the assay, similar in design, used the millimeter-scale surface area of an optical fiber (51). The later modification resulted in ultrasmall (100 $\mu \mathrm{m}$ to sub-micrometer size) DNA/RNA biosensors. The lower concentration limits of these biosensors were in the $0.3-10 \mathrm{nM}$ range of the target nucleic acid sequence (52).

\section{CONCLUSION}

DNA probes that use FRET detect many types of reactions involving DNA, RNA, proteins, and inorganic substances. Conformation-wise, the probes can be linear, hairpin-shaped, or have more complex configurations, as in the case of deoxyribozymes. The hairpinshaped probes represent one of the most popular designs. However, not only the probe configuration is important. The positioning, number, and nature of chromophores, as well as the incorporation of specific recognition and DNA enzyme sequences, are all components used in the construction of new probes.

An important aspect of FRET, making it an attractive technology in cellular research, is its ability to detect and monitor many of the reactions in live cells. It makes this approach a valuable tool for future in vivo visualization of cellular processes. Advantages of FRET DNA probes are also used in the new and quickly developing field of biosensors.

Another important application is the real-time monitoring of DNA amplification in PCR. Utilization of FRET permits the simultaneous detection of multiple products and is highly suitable for clinical diagnostic applications.

Progress in the field of energy transfer probes has recently resulted in the introduction of oligonucleotide probes, which contain nonfluorescent quenchers (89-91). In this case, the acceptor is nonfluorescent, and the probe reports detection of the target solely by the change in the fluorescence of the donor. The practical advantage is that the probe does not require the separation of the donor and acceptor fluorescence. These dark transfer reactions are based on contact quenching and do not need the spectral overlap specific for FRET $(90,91)$. The value of non-FRET-based quenching and the advantage of its combined usage with FRET were recognized in protein research relatively early $(2,12)$. However, DNA probes using the combination of FRET and nonFRET energy transfer mechanisms appeared only recently (91).

This novel direction in probe design represents a broader approach, leading to the development of principally new fluorescent oligonucleotide probes. These new constructs are not restrained by the limitations inherent to either FRET or contact quenching alone but instead combine their advantages in a more versatile energy transfer probe.

\section{ACKNOWLEDGMENTS}

This work was supported by the Baylor College of Medicine-Karolinska Institutet Research Exchange Program sponsored by the DeBakey Medical Foundation and by the grant no. 004949054 from the Texas Higher Education Coordinating Board. I thank Candace Minchew for technical assistance.

\section{REFERENCES}

1.Bazemore, R., M. Takahashi, and C.M. Radding. 1997. Kinetic analysis of pairing and strand exchange catalyzed by RecA. J. Biol. Chem. 272:14672-14682.

2.Bhattacharyya, A., B. Bhattacharyya, and S. Roy. 1993. A study of colchicine tubulin complex by donor quenching of fluorescence energy transfer. Eur. J. Biochem. 216:757761.

3.Biggins, J.B., J.R. Prudent, D.J. Marshall, M. Ruppen, and J.S. Thorson. 2000. A continuous assay for DNA cleavage: the application of "break lights" to enediynes, iron-dependent agents, and nucleases. Proc. Natl. Acad. Sci. USA 97:13537-13542.

4.Cardullo, R.A., S. Agrawal, C. Flores, P.C. Zamecnik, and D.E. Wolf. 1988. Nucleic acid hybridization by nonradiative fluorescence resonance energy transfer. Proc. Natl. Acad. Sci. USA 85:8790-8794.

5.Chen, X., K.J. Livak, and P.Y. Kwok. 1998. A homogeneous, ligase-mediated DNA diagnostic test. Genome Res. 8:549-556.

6.Chen, X. and P.Y. Kwok. 1997. Template-directed dye-terminator incorporation (TDI) assay: a homogeneous DNA diagnostic method based on fluorescence resonance energy transfer. Nucleic Acids Res. 25:347-353.

7.Chen, X. and P.Y. Kwok. 1999. Homogeneous genotyping assays for single nucleotide polymorphisms with fluorescence resonance energy transfer detection. Genet. Anal. 14:157-163.

8.Chen, X., B. Zehnbauer, A. Gnirke, and P.Y. Kwok. 1997. Fluorescence energy transfer detection as a homogeneous DNA diagnostic method. Proc. Natl. Acad. Sci. USA 94:10756-10761.

9.Clegg, R.M. 1995. Fluorescence energy transfer. Curr. Opin. Biotech. 6:103-110.

10.Clegg, R.M. 1992. Fluorescence resonance energy transfer and nucleic acids. Methods Enzymol. 211:353-388.

11.Cooper, J.P. and P.J. Hagerman. 1990. Analysis of fluorescence energy transfer in duplex and branched DNA molecules. Biochemistry 29:9261-9268.

12.Gryczynski, I., W. Wiczk, M.L. Johnson, H.C. Cheung, C.-K. Wang, and J.P. Lakow icz. 1988. Resolution of end-to-end distance distributions of flexible molecules using quenching-induced variations of the Forster distance for fluorescence energy transfer. Biophys. J. 54:577-586.

13.Damjanovich, S., R. Gaspar, and C. Pieri. 1997. Dynamic receptor superstructure at plasma membrane. Q. Rev. Biophys. 30:67106.

14.De Silva, D., A. Reiser, M. Herrmann, K. Tabit, and C. Wittwer. 1998. Rapid genotyping and quantification on the LightCycler ${ }^{\mathrm{TM}}$ with hybridization probes. Biochemica 2:1215.

15.Didenko, V.V., B. Zhang, H. Ngo, and D.S. Baskin. 1999. Detection of apoptotic DNA damage in live cells using fluorescence resonance energy transfer-development of molecular chameleons. Society for Neuroscience, Abstract.

16.Didenko V.V. and P.J. Hornsby. 1996. Pres- 
ence of double-strand breaks with $3^{\prime}$ singlebase overhangs in cells undergoing apoptosis but not necrosis. J. Cell Biol. 135:1369-1376.

17.Didenko V.V., J.R. Tunstead, and P.J. Hornsby. 1998. Biotin-labeled hairpin oligonucleotides: probes to detect doublestrand brakes in DNA in apoptotic cells. Am. J. Pathol. 152:897-902.

18.Didenko V.V., D.J. Boudreaux, and D.S. Baskin. 1999. Substantial background reduction in ligase-based apoptosis detection using newly designed hairpin oligoprobes. BioTechniques 27:1130-1132.

19.Doody, M.C., L.A. Sklar, H.J. Pownall, J.T. Sparrow, A.M. Gotto, Jr., and L.C. Smith. 1983. A simplified approach to resonance energy transfer in membranes, lipoproteins and spatially restricted systems. Biophys. Chem. 17:139-152.

20.Eftink, M.R. 1991. Fluorescence quenching: theory and applications, p. 53-126. In J.R. Lakowicz (Ed.), Topics in Fluorescence Spectroscopy, Vol. 2. Plenum Press, New York.

21.Fang, X., X. Liu, S. Scuster, and W. Tan. 1999. Designing a novel molecular beacon for surface immobilized DNA hybridization studies. J. Am. Chem. Soc. 121:2921-2922.

22.Förster, T. 1965. Delocalized excitation and excitation transfer, p. 93-137. In O. Sinanoglu (Ed.), Modern Quantum Chemistry, Istambu Lectures, part III. Academic Press, New York

23.Förster, T. 1946. Energiewanderung und Fluoreszenz. Naturwissenschaften 6:166-175

24.Förster, T. 1948. Zwischenmoleculare Energiewanderung und Fluoreszenz. Ann. Phys. (Leipzig) 2:55-75

25.Ghosh, S.S., P.S. Eis, K. Blumeyer, K. Fearon, and D.P. Millar. 1994. Real time kinetics of restriction endonuclease cleavage monitored by fluorescence resonance energy transfer. Nucleic Acids Res. 22:3155-3159.

26.Glazer, A.N. and R.A. Mathies. 1997. Energy-transfer fluorescent reagents for DNA analyses. Curr. Opin. Biotech. 8:94-102.

27.Gundry, C.N., P.S. Bernard, M.G. Hermann, G.H. Reed, and C.T. Wittwer. 1999 Rapid F508del and F508C assay using fluorescent hybridization probes. Genet. Test. 3:365-370

28.Gupta, R.C., E.I. Golub, M.S. Wold, and C.M. Radding. 1998. Polarity of DNA strand exchange promoted by recombination proteins of the RecA family. Proc. Natl. Acad. Sci. USA 95:9843-9848.

29.Gutierrez-Merino, C. 1981. Quantitation of the Förster energy transfer for two-dimensional systems, I. Lateral phase separation in unilamellar vesicles formed by binary phospholipid mixtures. Biophys. Chem. 14:247-257.

30.Gutierrez-Merino, C. 1981. Quantitation of the Förster energy transfer for two-dimensional systems, II. Protein distribution and aggregation state in biological membranes. Biophys. Chem. 14:259-266.

31.Hall, J.G., P.S. Eis, S.M. Law, L.R. Reynaldo, J.R. Prudent, D.J. Marshall, H.T. Allawi, A.L. Mast et al. 2000. Sensitive detection of DNA polymorphisms by the serial invasive signal amplification reaction. Proc. Natl. Acad. Sci. USA 97:8272-8277.

32.Heller, M.J. and L.E. Morrison. 1985. Chemiluminescent and fluorescent probes for
DNA hybridization, p. 245-256. In D.T. Kingsbury and S. Falkow (Eds.), Rapid Detection and Identification of Infectious Agents. Academic Press, New York.

33.Henry, M.R., P. Wilkins Stewens, J. Sun, and D.M. Kelso. 1999. Real-time measurements of DNA hybridization on microparticles with fluorescence resonance energy transfer. Anal. Biochem. 276:204-214.

34.Hessner, M.J., M.A. Budish, and K.D. Friedman. 2000. Genotyping of factor V G1691A (Leiden) without the use of PCR by invasive cleavage of oligonucleotide probes. Clin. Chem. 46:1051-1056.

35.Holland, P.M., R.D. Abramson, R. Watson, and D.H. Gelfand. 1991. Detection of specific polymerase chain reaction product by utilizing the $5^{\prime} \rightarrow 3^{\prime}$ exonuclease activity of Thermus aquaticus DNA polymerase. Proc. Natl. Acad. Sci. USA 88:7276-7280.

36.Hung, S.-C., J. Ju, R.A. Mathies, and A.N. Glazer. 1996. Energy transfer primers with 5or 6-carboxyrhodamine-6G as acceptor chromophores. Anal. Biochem. 238:165-170.

37.Hung, S.-C., J. Ju, R.A. Mathies, and A.N. Glazer. 1996. Cyanine dyes with high absorption cross-section as donor chromophores in energy transfer primers. Anal. Biochem. 243:15-27

38.Ju, J., C.C. Ruan, C.W. Fuller, A.N. Glazer, and R.A. Mathies. 1995. Fluorescence energy transfer dye-labeled primers for DNA sequencing and analysis. Proc. Natl. Acad. Sci. USA 92:4347-4351.

39.Ju, J., R.A. Mathies, and A.N. Glazer. 1996. Cassette labeling for facile construction of energy transfer fluorescent primers. Nucleic Acids Res. 24:1144-1148.

40.Ju, J., A.N. Glazer, and R.A. Mathies. 1996. Energy transfer primers-a new fluorescence labeling paradigm for DNA sequencing and analysis. Nat. Med. 2:246-249.

41.Kawahara, S., T. Uchimaru, and S. Murata. 1999. Efficiency enhancement of long-range energy transfer by sequential multistep FRET using fluorescence labeled DNA. Nucleic Acids Symp. Ser. 42:241-242.

42.Kostrikis, L.G., S. Tyagi, M.M. Mhlanga, D.D. Ho, and F.R. Kramer. 1998. Spectral genotyping of human alleles. Science 279:1228-1229.

43.Lakowicz, J.R. 1983. Quenching of fluorescence, p. 257-301. In Principles of Fluorescence Spectroscopy. Plenum Press, New York.

44.Lee, S.P., D. Porter, J.G. Chirikjian, J.R. Knutson, and M.K. Han. 1994. A fluorometric assay for DNA cleavage reactions characterized with $\mathrm{BamHI}$ restriction endonuclease. Anal. Biochem. 220:377-383.

45.Lee, S.P., M.L. Censullo, H.G. Kim, J.R. Knutson, and M.K. Han. 1995. Characterization of endonucleolytic activity of HIV-1 integrase using a fluorogenic substrate. Anal. Biochem. 227:295-301.

46.Lee, L.G., C.R. Connell, and W. Bloch. 1993. Allelic discrimination by nick-translation PCR with fluorogenic probes. Nucleic Acids Res. 21:3761-3766.

47.Li, J. and Y. Lu. 2000. A highly sensitive and selective catalytic DNA biosensor for lead ions. J. Am. Chem. Soc. 122:10466-10467.

48.Li, J.J., R. Geyer, and W. Tan. 2000. Using molecular beacons as a sensitive fluorescence assay for enzymatic cleavage of single-stranded DNA. Nucleic Acids Res. 28:E52.

49.Li, J., X. Fang, S. Schuster, and W. Tan. 2000. Molecular beacons: a novel approach to detect protein-nucleic acid interactions. Angew. Chem. Int. Ed. Engl. 39:1049-1052.

50.Lieber, M. 1997. The FEN-1 family of structure-specific nucleases in eukaryotic DNA replication, recombination and repair. BioEssays 19:233-240.

51.Liu, X. and W. Tan. 1999. A fiber-optic evanescent wave DNA biosensor based on novel molecular beacons. Anal. Chem. 71:5054-5059.

52.Liu, X., W. Farmerie, S. Schuster, and W. Tan. 2000. Molecular beacons for DNA biosensors with micrometer to submicrometer dimensions. Anal. Biochem. 283:56-63.

53.Livak, K.J., S.J.A. Flood, J. Marmaro, W. Giusti, and K. Deetz. 1995. Oligonucleotides with fluorescent dyes at opposite ends provide a quenched probe system useful for detecting PCR product and nucleic acid hybridization. PCR Methods Appl. 4:1-6.

54.Livak, K.J., J. Marmaro, and S. Flood 1995. Guidelines for designing Taqman fluorescent probes for $5^{\prime}$ nuclease assays. Research news. PE Applied Biosystems, Foster City, CA.

55.Loeffler, J., L. Hagmeyer, H. Hebart, N. Henke, U. Schumacher, and H. Einsele. 2000. Rapid detection of point mutations by fluorescence resonance energy transfer and probe melting curves in Candida species. Clin. Chem. 46:631-635.

56.Lyamichev, V., M.A.D. Brow, and J.E. Dahlberg. 1993. Structure-specific endonucleolytic cleavage of nucleic acids by eubacterial DNA polymerases. Science 260:778-783.

57.Lyamichev, V., A.L. Mast, J.G. Hall, J.R Prudent, M.W. Kaiser, T. Takova, R.W. Kwiatkowski, T.J. Sander et al. 1999. Polymorphism identification and quantitative detection of genomic DNA by invasive cleavage of oligonucleotide probes. Nat. Biotechnol. 17:292-296.

58.Maniatis, T., E.F. Fritsch, and J. Sambrook 1982. Molecular Cloning: A Laboratory Manual. CSH Laboratory Press, Cold Spring Harbor, New York.

59.Masuko, M., S. Ohuchi, K. Sode, H Ohtani, and A. Shimadzu. 2000. Fluorescence resonance energy transfer from pyrene to perylene labels fro nucleic acid hybridization assays under homogeneous solution conditions. Nucleic Acids Res. 28:E34.

60.Matko, J. and M. Edidin. 1997. Energy transfer methods for detecting molecular clusters on cell surfaces. Methods Enzymol. 278:444-462.

61.Matsuo, T. 1998. In situ visualization of messenger RNA for basic fibroblast growth factor in living cells. Biochim. Biophys. Acta 1379:178-184.

62.Medintz, I.L., C.C. Lee , W.W. Wong, K Pirkola, D. Sidransky, and R.A. Mathies. 2000. Loss of heterozygosity assay for molecular detection of cancer using energy-transfer primers and capillary array electrophoresis. Genome Res. 10:1211-1218.

63.Mein, C.A., B.J. Barratt, M.G. Dunn., T. 
Siegmund, A.N. Smith, L. Esposito, S. Nutland et al. 2000. Evaluation of single nucleotide polymorphism typing with Invader on PCR amplicons and its automation. Genome Res. 10:330-343.

64.Mergny, J.-L., A.S. Boutorine, T. Garestier, F. Belloc, M. Rougee, N.V. Bulychev, A.A. Koshkin, J. Bourson et al. 1994. Fluorescence energy transfer as a probe for nucleic acid structures and sequences. Nucleic Acids Res. 22:920-928.

65.Morrison, L.E., T.C. Halder, and L.M. Stols. 1989. Solution-phase detection of polynucleotides using interacting fluorescent labels and competitive hybridization. Anal. Biochem. 183:231-244.

66.Morrison, L.E. 1992. Detection of energy transfer and fluorescence quenching, p. 311352. In L.J. Kricka (Ed.), Nonisotopic DNA Probe Techniques. Academic press, San Diego.

67.Murakami, A., Y. Suminami, Y. Sakaguchi, S. Nawata, F. Numa, F. Kishi, and H. Kato. 2000. Specific detection and quantitation of SCC antigen 1 and SCC antigen 2 mRNAs by fluorescence-based asymmetric semi-nested reverse transcription PCR. Tumour Biol. $21: 224-234$

68.Nauck, M., H. Wieland, and W. Marz. 2000. Evaluation of the Roche Diagnostics LightCycler-Apo B 3500 Mutation Detection Kit. Clin. Chem. Lab. Med. 38:667-671.

69.Nauck, M., W. Marz, and H. Wieland. 2000. Evaluation of the Roche diagnostics LightCycler-Factor V Leiden Mutation Detection Kit and LightCycler-Prothrombin Mutation Detection Kit. Clin. Biochem. 33:213216.

70.Nazarenko I.A., S.K. Bhatnagar, and R.J. Hohman. 1997. A closed tube format for am plification and detection of DNA based on energy tranfer. Nucleic Acids Res. 25:25162521.

71.Neoh, S.H., M.J. Brisco, F.A. Firgaira, K.J. Trainor, D.R. Turner, and A.A. Morley. 1999. Rapid detection of the factor V Leiden $(1691 \mathrm{G}>\mathrm{A})$ and haemochromatosis (845 $\mathrm{G}>\mathrm{A}$ ) mutation by fluorescence resonance energy transfer (FRET) and real time PCR. J. Clin. Pathol. 52:766-769.

72. Okamura Y., S. Kondo, I. Sase, T. Suga, K. Mise, I. Furusawa, S. Kawakami, and Y. Watanabe. 2000. Double-labeled donor probe can enhance the signal of fluorescence resonance energy transfer (FRET) in detection of nucleic acid hybridization. Nucleic Acids Res. 28:E107.

73.Ortiz, E., G. Estrada, P.M. Lizardi. 1998. PNA molecular beacons for rapid detection of PCR amplicons. Mol. Cell. Probes 12:219-26.

74.Parkhurst, K.M. and L.J. Parkhurst. 1995. Donor-acceptor distance distribution in a double-labeled fluorescent oligonucleotide both as a single strand and in duplexes. Biochem istry 34:293-300

75.Piatek, A.S., S. Tyagi, A.C. Pol, A. Telenti, L.P. Miller, F.R. Kramer, and D. Alland. 1998. Molecular beacon sequence analysis for detecting drug resistance in Mycobacterium tuberculosis. Nat. Biotechnol. 16:359-363.

76. Rivetti, C., C. Walker, and C. Bustamante. 1998. Polymer chain statistics and conforma- tional analysis of DNA molecules with bends or sections of different flexibility. J. Mol. Biol. 280:41-59.

77.Rudert, W.A., E.R. Braun, S.J. Faas, R. Menon, A. Jaquins-Gerstl, and M. Trucco. 1997. Double-labeled fluorescent probes for 5 ' nuclease assays: purification and performance evaluation. BioTechniques 22:11401145

78.Sei-Iida, Y., H. Koshimoto, S. Kondo, and A. Tsuji. 2000. Real-time monitoring of in vitro transcriptional RNA synthesis using fluorescence resonance energy transfer. Nucleic Acids Res. 28:E59.

79.Selvin, P.R. 1995. Fluorescence resonance energy transfer. Methods Enzymol. 246:300334.

80.Shi, M.M. 2001. Enabling large-scale pharmacogenetic studies by high-throughput mutation detection and genotyping technologies. Clin. Chem. 47:164-172.

81.Sixou, S., F.C. Szoka, Jr., G.A. Green, B. Giusti, G. Zon, and D.J. Chin. 1994. Intracellular oligonucleotide hybridization detected by fluorescence resonance energy transfer (FRET). Nucleic Acids Res. 22:662-668.

82.Smith, L.M., J.Z. Sanders, R.J. Kaiser, P. Hughes, C. Dodd, C.R. Connell, C. Heiner, S.B.H. Kent et al. 1986. Fluorescence detection in automated DNA sequence analysis. Nature 321:674-679.

83.Snyder, B. and E. Freire. 1982. Fluorescence energy transfer in two dimensions: a numeric solution for random and nonrandom distributions. Biophys. J. 40:137-148.

84.Sokol, D.L., X. Zhang, P. Lu, and A.M. Gewirtz. 1998. Real time detection of DNA RNA hybridization in living cells. Proc. Natl. Acad. Sci. USA 95:11538-11543.

85.Stojanovic, M.N., P. de Prada, and D.W. Landry. 2000. Homogeneous assays based on deoxyribozyme catalysis. Nucleic Acids Res 28:2915-2918.

86.Stryer, L. 1978. Fluorescence energy transfer as a spectroscopic ruler. Annu. Rev. Biochem. 47:819-846.

87.Szöllosi, J., S. Damjanovich, and L. Matyus. 1998. Application of fluorescence resonance energy transfer in the clinical laboratory: routine and research. Cytometry 34:159-179.

88.Thelwell, N., S. Millington, A. Solinas, J. Booth, and T. Brown. 2000. Mode of action and application of Scorpion primers to mutation detection. Nucleic Acids Res. 28:37523761.

89.Tyagi, S. and F.R. Kramer. 1996. Molecular beacons-probes that fluoresce upon hybridization. Nat. Biotechnol. 14:303-308.

90.Tyagi, S., D. Bratu, and F.R. Kramer. 1998. Multicolor molecular beacons for allele discrimination. Nat. Biotechnol. 16:49.

91.Tyagi, S., S.A.E. Marras, and F.R. Kramer. 2000. Wavelength-shifting molecular beacons. Nat. Biotechnol. 18:1191-1196.

92.Uchiyama, H., K. Hirano, M. KashiwasakeJibu, and K. Taira. 1996. Detection of undegraded oligonucleotides in vivo by fluorescence resonance energy transfer. J. Biol. Chem. 271:380-384.

93.Uehara, H., G. Nardone, I. Nazarenko, and R.J. Hohman. 1999. Detection of telomerase activity utilizing energy transfer primers: comparison with gel- and ELISA-based detection. BioTechniques 26:552-558.

94.Vet, J.A., A.R. Majithia, S.A. Marras, S. Tyagi, S. Dube, B.J. Poiesz, and F.R. Kramer. 1999. Multiplex detection of four pathogenic retroviruses using molecular beacons. Proc. Natl. Acad. Sci. USA 96:63946399.

95.Wang, Y., J. Ju, B.A. Carpenter, J.M. Atherton, G.F. Sensabaugh, and R.A. Mathies. 1995. Rapid sizing of short tandem repeat alleles using capillary array electrophoresis and energy transfer fluorescent primers. Anal. Chem. 67:1197-1203.

96.Wang, Y., J.M. Wallin, J. Ju, G.F. Sensabaugh, and R.A. Mathies. 1996. High-resolution capillary array electrophoretic sizing of multiplexed short tandem repeat loci using energy-transfer fluorescent primers. Electrophoresis 17:1-6.

97.Whitcombe, D., J. Theaker, S.P. Guy, T. Brown, and S. Little. 1999. Detection of PCR products using self-probing amplicons and fluorescence. Nat. Biotechnol. 17:804807.

98. Wildeson, J. and C.J. Murphy. 2000. Intrinsic bending in GGCC tracts as probed by fluorescence resonance energy transfer. Anal. Biochem. 284:99-106.

99.Wittwer, C.T., K.M. Ririe, R.V. Andrew, D.A. David, R.A. Gundry, and U.J. Balis. 1997. The LightCycler ${ }^{\mathrm{TM}}$ : a microvolume multisample fluorimeter with rapid temperature control. BioTechniques 22:176-181.

100.Xu, D.-G. and T.M. Nordlund. 2000. Sequence dependence of energy transfer in DNA oligonucleotides. Biophys. J. 78:1042-1058.

Address correspondence to:

Dr. Vladimir V. Didenko

VAMC

2002 Holcombe Boulevard

Research Building 109, Room 204

Houston, TX 77030, USA

e-mail:vdidenko@bcm.tmc.edu

For reprints of this or any other article, contact Reprints@BioTechniques.com 